\title{
Prospects of Higgs portal DM models at future colliders
}

\author{
Jinmian $\mathbf{L i}^{*}$ \\ School of Physics, Korea Institute for Advanced Study, Seoul 130-722, Korea \\ E-mail: jmli@kias.re.kr \\ P. Ko \\ School of Physics, Korea Institute for Advanced Study, Seoul 130-722, Korea \\ E-mail: pko.kias.re.kr
}

\section{Bhaskar Dutta}

Mitchell Institute for Fundamental Physics and Astronomy, Department of Physics and Astronomy, Texas A\&M University, College Station, TX 77843-4242, USA

E-mail:dutta@physics.tamu.edu

\section{Teruki Kamon}

Mitchell Institute for Fundamental Physics and Astronomy, Department of Physics and Astronomy, Texas A\&M University, College Station, TX 77843-4242, USA

E-mail:kamon@physics.tamu.edu

\begin{abstract}
We study the discovery and discriminating prospects of the Higgs portal dark matter (DM) models for scalar, fermion and vector DM at the ILC with $\sqrt{s}=500 \mathrm{GeV}$ and at proton-proton (pp) collider with $\sqrt{s}=100 \mathrm{TeV}$. For the singlet scalar DM, the mediator is just the Standard Model (SM) Higgs boson, whereas for the fermion or vector DM there is an additional singlet scalar mediator that mixes with the SM Higgs boson, which produces significant observable differences. At the $500 \mathrm{GeV}$ ILC, the DM pair is dominantly produced in association with a $\mathrm{Z}$ boson. The distributions of DM pair invariant mass can be reconstructed. It is used as the main handle for discriminating the DM spin. At the $100 \mathrm{TeV} p$ - $p$ collider, we consider the $t \bar{t}+\mathrm{DM}$ associated production in dileptonic final states. The two dimensional distributions of missing transverse energy and polar angle difference between two leptons are used for a discrimination of the spin nature of DM.
\end{abstract}

The 39th International Conference on High Energy Physics (ICHEP2018)

4-11 July, 2018

Seoul, Korea

${ }^{*}$ Speaker. 


\section{Introduction}

The DM effective field theory is found to be only valid for the process where the momentum transfer is much smaller than the mass of the mediator such as in DM direct detection experiments. Meanwhile, the DM simplified model also suffers from the breaking of the SM gauge symmetry, violating of unitarity as well as lacking some important gauge invariant interactions. It is important and more realistic to consider the DM search at the collider in the framework of UV-complete gauge invariant and renormalizable models. This work aims to study the discovery as well as the discriminating prospects of Higgs portal dark matter (DM) models for scalar, fermion and vector DM both at the future linear collider [1] and future high energy proton-proton collider [2].

\section{The gauge invariant models for scalar, fermion and vector DM}

The scalar DM (SDM )model can be constructed by introducing a new scalar $S$ to the SM

$$
\mathscr{L}_{\text {SDM }}=\frac{1}{2} \partial_{\mu} S \partial^{\mu} S-\frac{1}{2} m_{0}^{2} S^{2}-\lambda_{H S} H^{\dagger} H S^{2}-\frac{\lambda S}{4 !} S^{4},
$$

where $H$ is the SM Higgs doublet and $S$ is assumed to be odd under a $Z_{2}$ symmetry and thus becomes a DM candidate. After the electroweak (EW) symmetry breaking $H \rightarrow\left(0,\left(v_{h}+h\right) / \sqrt{2}\right)^{T}$ and assuming $\langle S\rangle=0$, we can write down the interaction Lagrangian for DM production at the ILC as

$$
\mathscr{L}_{\mathrm{SDM}}^{\mathrm{int}}=-h\left(\frac{2 m_{W}^{2}}{v_{h}} W_{\mu}^{+} W^{-\mu}+\frac{m_{Z}^{2}}{v_{h}} Z_{\mu} Z^{\mu}\right)-\lambda_{H S} v_{h} h S^{2} .
$$

In this model, the DM can only be pair produced through the SM Higgs ( $h$ ) mediation.

The simplest Higgs portal singlet fermion DM (FDM) model with SM gauge invariance contains a SM singlet Dirac fermion DM $\chi$ and a real singlet scalar mediator $S$ :

$$
\begin{aligned}
\mathscr{L}_{\mathrm{FDM}} & =\bar{\chi}\left(i \not \partial-m_{\chi}-y_{\chi} S\right) \chi+\frac{1}{2} \partial_{\mu} S \partial^{\mu} S-\frac{1}{2} m_{0}^{2} S^{2} \\
& -\lambda_{H S} H^{\dagger} H S^{2}-\mu_{H S} S H^{\dagger} H-\mu_{0}^{3} S-\frac{\mu_{S}}{3 !} S^{3}-\frac{\lambda_{S}}{4 !} S^{4},
\end{aligned}
$$

where the singlet Dirac fermion $\chi$ is assumed to be odd under a $Z_{2}$ dark parity $\chi \rightarrow-\chi$. When both scalar fields $H$ and $S$ develop nonzero vacuum expectation values (VEV), $v_{h}$ and $v_{s}$, the two scalar fields mix, giving $H_{1}$ and $H_{2}$ fields in mass eigenstate. The interaction Lagrangian of interest can be written in the mass eigenstates as

$$
\begin{aligned}
\mathscr{L}_{\mathrm{FDM}}^{\mathrm{int}} & =-\left(H_{1} \cos \alpha+H_{2} \sin \alpha\right)\left(\sum_{f} \frac{m_{f}}{v_{h}} \bar{f} f-\frac{2 m_{W}^{2}}{v_{h}} W_{\mu}^{+} W^{-\mu}-\frac{m_{Z}^{2}}{v_{h}} Z_{\mu} Z^{\mu}\right) \\
& +g_{\chi}\left(H_{1} \sin \alpha-H_{2} \cos \alpha\right) \bar{\chi} \chi .
\end{aligned}
$$

As for constructing a renormalizable and gauge invariant model for vector (VDM), we need to introduce an abelian dark gauge group $U(1)_{X}$ and a dark Higgs field $\Phi$ :

$$
\mathscr{L}_{\mathrm{VDM}}=-\frac{1}{4} V_{\mu \nu} V^{\mu \nu}+D_{\mu} \Phi^{\dagger} D^{\mu} \Phi-\lambda_{\Phi}\left(\Phi^{\dagger} \Phi-\frac{v_{\phi}^{2}}{2}\right)^{2}-\lambda_{H \Phi}\left(H^{\dagger} H-\frac{v_{h}^{2}}{2}\right)\left(\Phi^{\dagger} \Phi-\frac{v_{\phi}^{2}}{2}\right),
$$


where the VEV of $\Phi=\frac{1}{\sqrt{2}}\left(v_{\phi}+\phi\right)$ will provide mass to the vector DM $V_{\mu}$. In this model, a $Z_{2}$ symmetry $\left(V_{\mu} \rightarrow-V_{\mu}\right)$ and charge conjugation symmetry have been imposed by hand, thereby forbidding the kinetic mixing between $V_{\mu}$ and the SM $U(1)_{Y}$ gauge boson and making the vector boson $V_{\mu}$ stable. The interaction Lagrangian that is relevant to the collider study can be written as

$$
\begin{aligned}
\mathscr{L}_{\mathrm{VDM}}^{\mathrm{int}} & =-\left(H_{1} \cos \alpha+H_{2} \sin \alpha\right)\left(\sum_{f} \frac{m_{f}}{v_{h}} \bar{f} f-\frac{2 m_{W}^{2}}{v_{h}} W_{\mu}^{+} W^{-\mu}-\frac{m_{Z}^{2}}{v_{h}} Z_{\mu} Z^{\mu}\right) \\
& -\frac{1}{2} g_{V} m_{V}\left(H_{1} \sin \alpha-H_{2} \cos \alpha\right) V_{\mu} V^{\mu} .
\end{aligned}
$$

\section{The prospects of searches at future colliders}

In order to guarantee sufficient DM production rate at colliders while consistent with current measurements, the relevant parameters for the fermion DM production are chosen as: $g_{\chi}=$ $3, \sin \alpha=0.3, m_{\chi}=80 \mathrm{GeV}$. Four benchmark points with different $m_{H_{2}}=(200,300,400,500)$ $\mathrm{GeV}$ will be studied, which are denoted as FDM200, FDM300, FDM400 and FDM500, respectively. For each benchmark point, we assume that the decay width for heavier scalar $H_{2}$ into the $H_{1}$ pair is negligible. In study the spin discrimination, the parameters for the vector DM production are chosen accordingly, i.e. $\sin \alpha=0.3, m_{V}=80 \mathrm{GeV}$ and the $g_{V}$ is chosen such that the total decay width of $H_{2}$ is the same with that in the fermion DM case, since one can rely on other method to measure the total decay width of $H_{2}$. The scalar DM model only includes two parameters: $m_{S}$ and $\lambda_{H S}$. We will fix $m_{S}=80 \mathrm{GeV}$ and take appropriate $\lambda_{H S}$ such that the number of signal events after all selections are kept the same as that of each benchmark point of the FDM model.

\subsection{At $500 \mathrm{GeV}$ linear collider}

The DM signal process at the ILC is $e^{+} e^{-} \rightarrow Z H_{1 / 2}(\rightarrow D D)$, where $D=S, \chi, V_{\mu}$ for scalar, fermion and vector DM, respectively. The hadronic decay mode of $Z$ boson will be considered, due to its larger branching ratio. The dominant SM backgrounds are $Z(\rightarrow j j)+v_{e} \bar{v}_{e}$ and $Z(\rightarrow j j) Z(\rightarrow$ $v v)$. To separate signal and background events, we first apply the preselections: (1) no leptons with $p_{T}(\ell)>10 \mathrm{GeV},|\eta(\ell)|<2.4$ in the final state; (2) exactly two $R=0.5$ anti- $k_{t}$ jets with $p_{T}(j)>20$ $\mathrm{GeV}$ and $|\eta|<3.0$ in the final state; (3) $E_{T}^{\text {miss }} \equiv\left|p_{T}^{\text {miss }}\right|>50 \mathrm{GeV}$. Then, the boosted decision tree method with inputs of $m_{D D}, E_{T}^{\text {miss }}, p_{T}(Z), p_{T}\left(j_{1}\right), \Delta \phi^{\min }=\min _{i=1,2} \Delta \phi\left(p_{T}^{\text {miss }}, p\left(j_{i}\right)\right), p_{T}\left(j_{2}\right)$ and $m_{j j}$ are adopted to further improve the signal-to-background ratio. The cuts flow of the analysis is given in Tab. 1.

\subsection{At $100 \mathrm{TeV} p$ - $p$ collider}

The DM signal at the $100 \mathrm{TeV} p-p$ collider that we consider is $t \bar{t}+\mathrm{DM}$ associated production, with both top quark decaying leptonically. The main backgrounds are SM $t \bar{t}, t \bar{t} W$ and $t \bar{t} Z$. The selections cuts are adopted as follows: (1) exactly two opposite sign leptons with $p_{T}>20 \mathrm{GeV}$ and $|\eta|<2.5$; (2) at least one $\mathrm{R}=0.5$ anti- $k_{t} b$ jet with $p_{T}>25 \mathrm{GeV}$ and $|\eta|<2.5$; (3) $m_{\ell \ell} \notin[85,95]$ $\mathrm{GeV}$; (4) $E_{T}^{\text {miss }}>150 \mathrm{GeV}$; (5) $m_{T_{2}}(\ell, \ell)>150 \mathrm{GeV}$. The discovery prospects of our benchmark points with varying integrated luminosity is given in the left panel of Fig. 1. Next, we use the two dimensional distributions of $\cos \left(\theta_{\ell \ell}\right)$ and $E_{T}^{\text {miss }}$ for distinguishing FDM and VDM, FDM and SDM. 


\begin{tabular}{|c||c|c|c|c|}
\hline & FDM200 & FDM300 & FDM400 & FDM500 \\
\hline$\sigma^{0}[\mathrm{fb}]$ & 1.643 & 0.9214 & 0.4221 & 0.2526 \\
\hline$\varepsilon^{\text {pre }}$ & 0.796 & 0.717 & 0.655 & 0.698 \\
\hline BDT & 0.3615 & 0.2132 & 0.1929 & 0.2129 \\
\hline$N_{S} / 1000 \mathrm{fb}^{-1}$ & 697.8 & 410.5 & 148 & 102 \\
\hline$N_{B} / 1000 \mathrm{fb}^{-1}$ & 2248.5 & 11453.5 & 12736 & 10898 \\
\hline$N_{S} / \sqrt{N_{S}+N_{B}}$ & 12.85 & 3.769 & 1.31 & 0.97 \\
\hline
\end{tabular}

Table 1: The total production cross section $\left(\sigma^{0}\right)$, cross section after pre-selection $\left(\varepsilon^{\text {pre }}\right)$, the chosen BDT cut (BDT), number of signal $\left(N_{S}\right)$ and background $\left(N_{B}\right)$ events after BDT cut and the signal significance $\left(N_{S} / \sqrt{N_{S}+N_{B}}\right)$ at the ILC with $\sqrt{s}=500 \mathrm{GeV}$ and $\mathscr{L}=1000 \mathrm{fb}^{-1}$ for benchmark points in FDM model.

We adopt the binned likelihood test with $\mathscr{L}\left(\right.$ data $\left.\mid \mathscr{H}_{\alpha}\right)=\prod_{i, j} \frac{{ }_{i j}^{n_{i j}} e^{-t_{i j}}}{n_{i j} !}$ to evaluate the $p$-value in the discrimination, where $\mathscr{H}_{\alpha}$ is the hypothesis of DM spin. The discrimination power with varying integrated luminosity is given the middle and right panel of Fig. 1.
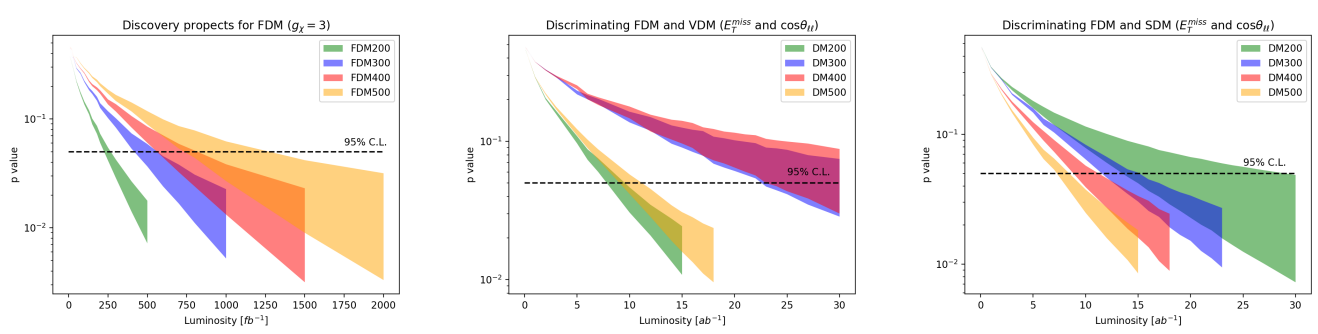

Figure 1: Discovery prospects for fermion DM (left). Spin discrimination prospects between FDM and VDM (middle); between FDM and SDM (right). The widths of the bands correspond to the effects of systematic uncertainty, e.g. $1 \%$ for discovery and $0.5 \%$ for spin discrimination.

\section{Conclusion}

The gauge invariant Higgs portal DM models for FDM and VDM require at least two scalar mediators, while that of SDM only need one. At the ILC, benchmark points $m_{H_{2}} \lesssim 300 \mathrm{GeV}$ can be probed at more than 3- $\sigma$ level. At the $100 \mathrm{TeV} p-p$ collider, discovery/exclusion can be made with an integrated luminosity less than $1 \mathrm{ab}^{-1}$ given a $1 \%$ systematic uncertainty for all benchmark points. The spin discrimination requires integrated luminosity of a few $\mathscr{O}(10) \mathrm{ab}^{-1}$ given a $0.5 \%$ systematic uncertainty.

\section{References}

[1] T. Kamon, P. Ko and J. Li, Eur. Phys. J. C 77, no. 9, 652 (2017) doi:10.1140/epjc/s10052-017-5240-8 [arXiv:1705.02149 [hep-ph]].

[2] B. Dutta, T. Kamon, P. Ko and J. Li, Eur. Phys. J. C 78, no. 7, 595 (2018) doi:10.1140/epjc/s10052-018-6071-y [arXiv:1712.05123 [hep-ph]]. 